\title{
Editorial
}

\section{THYROID SURGERY: THE DOMAIN OF WHOM?}

This is an abstract that I had prepared in 2001 for a conference in Birmingham, UK. The issues discussed here are still relevant to thyroid surgery.

Thyroid surgery interests general surgeons, otolaryngologists, surgical oncologists, head and neck surgeons and pediatric surgeons. Thyroid surgery used to be the domain of general surgeons and most of the surgeons from the early part of the 20th century hailed from general surgery background. It was considered to be the domain of the general surgeons up until the last two decades. With an overall lack of interest in head and neck surgery from general surgeons and their

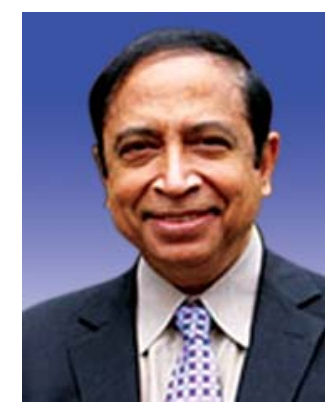
increasing interest in endoscopic and vascular surgery, the field of thyroid surgery was left open to be encroached upon by surgeons from other specialties and interests. Clearly, the specialties, such as otolaryngology and well-trained otolaryngologists seized the opportunity to expand their horizons and widen their surgical field and surgical expertize to include thyroid surgery. O bviously, the otolaryngologists that routinely performed laryngectomy did not have any problem in learning and mastering the technique of thyroid surgery. However, their understanding of the thyroid physiology, diagnostic workup and the follow-up of patients with benign and malignant thyroid diseases was not complete. The dominant issues relate to a variety of factors, such as financial remuneration, institutional policy, the departmental guidelines, the perceived complication rate, and the expertize available in other surgical specialties.

A majority of the head and neck surgery in the $U$ nited States has been shifted from the hands of the general surgeons to the otolaryngologists. Unfortunately, this has probably led to the false belief by every otolaryngologist that he or she is a head and neck surgeon, qualified to perform major head and neck surgery. In the last two decades, the majority of otolaryngology divisions have become departments. Such turf issues are related not only to thyroid surgery but also many other surgical procedures, such as vascular surgery, carotid endarterectomy, pituitary surgery, etc.

Thyroid surgery is one of the most common surgical procedure and it generates considerable debate and controversy. A lthough the domain issue may not be resolved, I strongly feel that thyroid surgery should be performed by those who understand both the diagnostic workup and the principles in the management of benign and malignant thyroid tumors, including the management of complications and the long-term treatment of thyroid cancer.

W ith the current shift in head and neck surgery from general surgeon to otolaryngologist, we are going to see more and more well-trained surgeons with an otolaryngology background who are interested and well qual ified in the management of thyroid cancer. Clearly, the parent subspecialty strengthens some of the issues in thyroid surgery, such as appropriate evaluation of the vocal cord and larynx, understanding of the disease invading or approaching the trachea and esophagus, and the understanding and management of vocal cord paralysis. The issues related to thyroid surgery should also be critically analyzed in view of the complications related to thyroid surgery, such as hypoparathyroidism and vocal cord injury.

In addition, there should be a standardized audit every year in each institution. Udelsman et al have shown that the thyroid/parathyroid surgery performed in specialized centers has a much lower incidence of major complications. Thus, it is extremely important to have a cooperative group of physicians taking care of thyroid and parathyroid disease, including endocrinologists, nuclear medicine physicians, radiologists and surgeons. Even though one may not be able to answer the issue related to domain, it is clear that thyroid surgery should be performed by a well-trained surgeon who understands the technical details, the complications and their management, and the follow-up for patients with thyroid cancer.

Thyroid surgery, whose domain is it? I am not sure we can find an answer to this question, but I would like to summarize. Thyroid surgery is the domain of the well-trained thyroid surgeon irrespective of the background specialty. It should not be the domain of the parent specialty, but the domain of a well-trained thyroid surgeon.

Ashok R Shaha MD, FACS

Professor of Surgery, Jatin P Shah Chair in Head and Neck Surgery Memorial Sloan-Kettering Cancer Center, New York, USA Phone: 212-639-7649, Fax: 212-717-3302 e-mail: shahaa@mskcc.org 